\title{
NÍVEIS DE REGENERAÇÃO NATURAL EM FLORESTA DE TERRA FIRME NO AMAPÁ BRASIL ${ }^{1}$
}

Perseu Silva Aparicio², Eleneide Doff Sotta ${ }^{3}$, Marcelino Carneiro Guedes ${ }^{3}$, Wegliane Campelo da Silva Aparício $^{4}$, Lana Patricia Oliveira ${ }^{5}$ e Raianny Nayara Souza ${ }^{6}$

\begin{abstract}
RESUMO - Este trabalho objetivou identificar a regeneração natural na fitofisionomia ombrófila densa da Floresta Estadual do Amapá, descrevendo as relações entre estratos verticais. A área de estudo está localizada no Município de Porto Grande, AP, Amazônia oriental. Foram implantados três conglomerados, equidistantes $2.500 \mathrm{~m}$, para a estimativa da regeneração natural. Foram utilizadas 100 subparcelas de $10 \times 10 \mathrm{~m}$ para o estudo das arvoretas (5,0< dap (diâmetro a 1,30 $\mathrm{m}$ do solo) $<10,0 \mathrm{~cm})$ e varas $(5$ x $5 \mathrm{~m})(2,5<$ dap $<5,0 \mathrm{~cm}$ ) por conglomerado. Coletaram-se as alturas e nomes populares. As alturas foram divididas em três classes de regeneração, para a estimativa dos parâmetros fitossociológicos de frequência e densidade, bem como da regeneração por classe de tamanho e total. A diversidade foi estimada pelo Índice de Shannon. Também, analisouse a similaridade florística entre as varas e arvoretas por conglomerado. O inventário contemplou 2.700 indivíduos, pertencentes a 38 famílias botânicas, 93 gêneros e 141 espécies arbóreas, com seis indeterminadas. O índice de Shannon foi de 4,21 (arvoretas) e 4,11 nats.ind. ${ }^{-1}$ (varas). Foi constatada a ocorrência de 33 espécies comuns nas três classes de regeneração. A regeneração natural total apresentou variações de 10,3 a 0,1\% (varas) e 5,6 a 0,1\% (arvoretas). As espécies com menores percentuais foram as Indeterminadas, Vouacapoua americana, Carapa guianensis, Virola calophylla e Manilkara huberi. Logo, fica evidente que as espécies estão desenvolvendo seu processo sucessional de forma eficiente, garantindo a conservação da fitofisionomia da região.
\end{abstract}

Palavras-chave: Classes de altura; Similaridade florística; Métodos de amostragem.

\section{LEVELS OF NATURAL REGENERATION IN DRY LAND FOREST IN AMAPA BRAZIL}

\begin{abstract}
This study aimed to identify natural regeneration in dense ombrophilous phytophysiognomy of the State Forest of Amapá, describing relations between vertical stratums. The study area is located in Porto Grande/AP, eastern Amazon. Three conglomerates were implanted, equidistant at $2.500 \mathrm{~m}$, for natural regeneration estimative. We used 100 plots of $10 \times 10 \mathrm{~m}$ for study of saplings (5.0<DBH (diameter at $1.30 \mathrm{~m}$ soil $)<10.0 \mathrm{~cm})$ and sticks $(5 \times 5 \mathrm{~m})(2.5<\mathrm{DBH}<5.0 \mathrm{~cm})$ in each conclomerate. We collected the heights and popular names. The heights were divided into three classes of regeneration to estimate phytosociological parameters of frequency and density, as well as regeneration by size class and overall. Diversity was estimated by Shannon Index. Floristic similarity between sticks and saplings was also analyzed. The inventory included 2,700 individuals belonging to 38 botanical families, 93 genera and 141 species of trees, with 6 undetermined. The Shannon index was 4.21 (saplings) and 4.11 nats.ind.-1 (sticks). It has been found the occurrence of 33 common species in three classes of regeneration. The total natural regeneration varied from 10.3 to $0.1 \%$ (sticks) and 5.6 to $0.1 \%$ (saplings). The species with the lowest percentages were Indeterminate, Vouacapoua americana, Carapa guianensis, Virola calophylla and Manilkara huberi. Thus, it is evident that species are developing their successional process efficiently, ensuring conservation of phytophysiognomy in the region.
\end{abstract}

Keywords: Height classes; Floristic similarity; Sampling methods.

\footnotetext{
${ }^{1}$ Recebido em 29.06.2013 aceito para publicação em 27.05.2014

${ }^{2}$ Departamento de Engenharia Florestal, Universidade do Estado do Amapá, UEAP, Brasil. E-mail: <perseu.aparicio@ueap.edu.br>.

${ }^{3}$ Empresa Brasileira de Pesquisa Agropecuária, EMBRAPA, Brasil. E-mail: <esotta@cpafap.embrapa.br> e <mcguedes@cpafap.embrapa.br>.

${ }^{4}$ Universidade Federal do Amapá, UNIFAP, Brasil. E-mail: <wellaparicio@unifap.br>.

${ }^{5}$ Mestre em Desenvolvimento Regional, Universidade Federal do Amapá, UNIFAP, Brasil. E-mail: <Lana_p_oliveira@hotmail.com>.

${ }^{6}$ Bolsista de Iniciação científica/CNPq, Universidade do Estado do Amapá, UEAP, Brasil. E-mail: <raiannynayara@hotmail.com>.
} 


\section{INTRODUÇÃO}

A exuberância e fragilidade dos ambientes na Amazônia são alvos de inúmeras discussões na sociedade, sejam para utilização dos recursos, seja para preservação dos ecossistemas. No caso das florestas, a composição e distribuição das árvores, principalmente dos indivíduos em fase de regeneração natural, orientam o desenvolvimento dos temas sobre sustentabilidade.

Atualmente, a sustentabilidade das atividades de exploração nas florestas tropicais é questionada devido ao fato de a quantidade retirada de madeira ocorrer sem considerar a peculiaridade de cada sítio. A legislação brasileira permite nas florestas primitivas da Amazônia a retirada de até $30 \mathrm{~m}^{3}$ a cada hectare, prevista na Instrução Normativa MMA 05/2006. Entretanto, o estabelecimento da regeneração natural varia de acordo com as condições de cada sítio e das operações de manejo.

Diante dessa problemática, caracterizar o estado da regeneração natural em florestas tropicais da Amazônia é fundamental para inferir sobre o estado de conservação e estoque disponível de espécies para garantir a resiliência do meio. Tal prerrogativa é fundamentada na teoria de que a regeneração natural representa o conjunto de indivíduos capazes de ser recrutados para os estádios posteriores (GAMA et al., 2003; SILVA et al., 2007).

De acordo com Martins e Rodrigues (2002), é preciso caracterizar o momento de estabelecimento das espécies na floresta para definir estratégias de conservação. A maneira mais tradicional de caracterizar as espécies regenerantes é por meio de amostras quali-quantitativas na floresta. Dessa maneira, as informações permitem reconhecer a função das espécies na comunidade, bem como suas preferências em hábitat e relações ecológicas inter e intraespecíficas.

Nesses termos, uma avaliação momentânea da regeneração natural subsidia a predição da estrutura da floresta adulta. Assim, a garantia da permanência de determinada espécie em uma floresta é função direta do número de indivíduos e da distribuição nas classes de tamanho, baseados no diâmetro ou na altura.

Em florestas ombrófila densas da Amazônia, estudos apontam alta diversidade de espécies arbóreas em fase de regeneração, porém com grande percentual de espécies representadas apenas por um indivíduo (PRANCE et al., 1976; LIMAFILHO, 2001; APARÍCIO, 2011a). No Amapá, o desenvolvimento de uma economia sustentável baseada nas pretensões de destinar a Floresta Estadual a concessões é limitado pela carência de informações técnicas sobre o estado das espécies arbóreas nas distintas fases de crescimento.

Esse cenário tem despertado o interesse da sociedade científica para desenvolver pesquisas que possam garantir a conservação das espécies após o início das atividades exploratórias na Floresta Estadual. Logo, para obter respostas que fortaleçam a eficiência da implantação do manejo florestal sustentável, este trabalho tentou responder à seguinte questão: A estrutura das espécies arbóreas regenerantes garante significativa representatividade para compor o futuro dossel da florestal?

Diante do exposto, o objetivo foi verificar como ocorre a regeneração natural na fitofisionomia ombrófila densa de terras baixas da Floresta Estadual do Amapá, descrevendo as relações entre estratos verticais.

\section{MATERIAL E MÉTODOS}

O trabalho foi realizado na Floresta Estadual do Amapá (FLOTA/AP), no Município de Porto Grande, região central do Estado do Amapá, Amazônia oriental, Brasil. A vegetação é perenifólia, característica da fitofisionomia ombrófila densa de terras baixas (IBGE, 2012). O solo predominante é do tipo Latossolo VermelhoAmarelo distrófico. Adeclividade característica é ondulada, com altitude variando de 60 a 100 m (RADAM, 1974).

O inventário florestal foi realizado em três conglomerados, equidistantes em aproximadamente 2.500 m. Cada conglomerado foi constituído por cinco parcelas permanentes de 100 x 100 m, equidistantes em 250 m.

Para estimativa da regeneração natural, cada parcela foi dividida em 100 subparcelas de 10 x 10 m. Dessas, foram selecionadas aleatoriamente 20 subparcelas/parcela para avaliação das arvoretas. Nos vértices das subparcelas sorteadas, foram locadas, aleatoriamente, subunidades de 5 x 5 m para estimativas das varas. O critério de inclusão se baseou a partir das classes de tamanho utilizadas por Silva et al. (2005), em que os indivíduos são classificados de acordo com os diâmetros: arvoretas (5,0 $\leq$ dap (diâmetro a 1,30 m do solo) < 10,0 cm) e varas $(2,5 \leq$ dap $<5,0 \mathrm{~cm})$ (Figura 1).

Dos indivíduos amostrados, foram registrados o nome popular e a altura comercial. A sinonímia e a grafia dos taxa foram atualizadas mediante a consulta ao índice de 


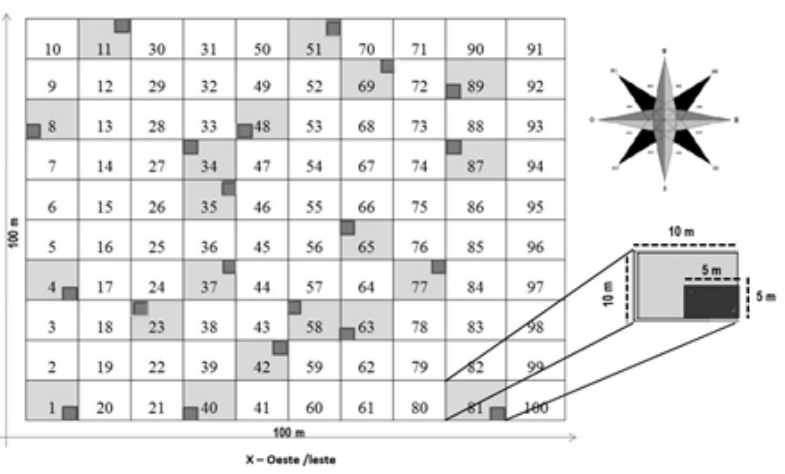

Figura 1 - Unidade Amostral Secundária (US) permanente, utilizada para o estudo das espécies arbóreas adultas e em regeneração natural, no segundo módulo da FLOTA/AP.

Figure 1 - Secondary Sampling Unit (U.S.) permanently used for the study of adult tree species and natural regeneration in the second module of FLOTA/AP.

espécies do Royal Botanic Garden e do banco de dados do Missouri Botanical Garden, disponível na página http:/ /mobot.mobot.org/W3T/Search/vast.html. O sistema de classificação adotado foi o APG II (2003). Para representar a regeneração natural como estrutura vertical dos indivíduos que se apresentaram com diâmetro mínimo exigido no critério de inclusão, as alturas $(\mathrm{H})$ foram divididas em classes de tamanho, em que a classe I contemplou indivíduos de 1,0 $\leq \mathrm{H}<5,0 \mathrm{~m}$; classe II, com indivíduos de 5,0 $\leq$ $\mathrm{H}<10,0 \mathrm{~m}$; e a classe III, com indivíduos $\geq 10,0 \mathrm{~m}$.

As análises dos parâmetros fitossociológicos foram realizadas para as classes de tamanho de acordo com Finol (1971). Além disso, quantificados o número de indivíduos por espécie nas distintas classes de tamanho, também foi estimada a diversidade de Shannon (H') em cada estrato (ODUM, 1988). A similaridade florística entre as classes de tamanho de arvoretas e varas nos conglomerados foi realizada por meio de matriz de presença e ausência das espécies, nos níveis de regeneração natural. Posteriormente, essa matriz foi utilizada para análise de agrupamento (ligação simples), tendo como medida a distância euclidiana.

\section{RESULTADOS}

O número total de indivíduos amostrados foi de 2.700 plantas vivas, pertencentes a 38 famílias botânicas, 93 gêneros e 141 espécies arbóreas, com seis indeterminadas. Quando consideradas apenas as arvoretas, foram inventariados 1.770 indivíduos, pertencentes a 30 famílias, 126 espécies e quatro indeterminadas. No caso dos indivíduos em nível de vara, foram inventariados 919 indivíduos e quantificadas 29 famílias, 111 espécies e cinco indeterminadas.

O índice de diversidade de Shannon ( $\left.\mathrm{H}^{\prime}\right)$ encontrado foi de 4,21 nats/ind., para a regeneração natural em arvoreta; e 4,11 nats/ind. em nível de vara. A diversidade de espécies foi mais bem representada entre as famílias com maior riqueza: Fabaceae (33 espécies), Sapotaceae (11), Lauraceae (10), Burseraceae (8) e Chrysobalanaceae (6).

As espécies que se destacaram com maior número de indivíduos foram, respectivamente: Hirtella sp. (139 plantas), Eschweilera coriaceae (117 plantas), Mouriri apiranga (116), Protium tenuifolium (112) e Guatteria sp. (99).

Os parâmetros fitossociológicos relativos demonstraram que a composição florística e a distribuição das espécies se modificam à medida que as plantas vão atingindo o dossel imediatamente superior. Quanto à frequência relativa, observou-se que o número de espécies reduziu com o aumento das alturas das plantas, tanto para arvoretas quanto para varas. As arvoretas apresentaram 48 (classe I), 44 (classe II) e 35 (classe III) espécies. Nas varas, foi observada menor riqueza, com 38 espécies na classe I, 37 na classe II e apenas quatro na classe III.

As espécies que se destacaram com densidades relativas elevadas nas classes de regeneração natural foram: Eugenia sp. e Hirtella sp. (5,3\%), Mouriri apiranga (4,2\%) (arvoretas), Mouriri apiranga e Hirtella sp. (varas) para classe I; Eschweilera coriacea e Protium tenuifolium (arvoretas), Guatteria sp. e Pourouma ovata (varas) para classe II; e destaque apenas para a Protium tenuifolium, tanto para arvoreta quanto para vara, na classe III.

A distribuição das arvoretas em regeneração evidenciou que 665 indivíduos, em 100 espécies, estão presentes na classe de altura I, seguida da classe de altura II (1.056 indivíduos pertencentes a 115 espécies) e da classe de altura III, com 49 indivíduos de 33 espécies.

As espécies inventariadas em nível de arvoreta (126) apresentaram 33 táxons presentes em todas as classes de altura. Os percentuais de arvoretas presentes nas classes de regeneração foram, principalmente, da Hirtella sp. e Eschweilera coriacea (classes I e II) e Protium tenuifolium (classe III). A distribuição percentual das arvoretas nas classes demonstrou que $41,12 \%$ delas estavam na classe I, seguida da classe II $(40,77 \%)$ e da classe III (18,09\%) (Tabela 1$)$.

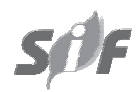

Revista Árvore, Viçosa-MG, v.38, n.4, p.699-710, 2014 


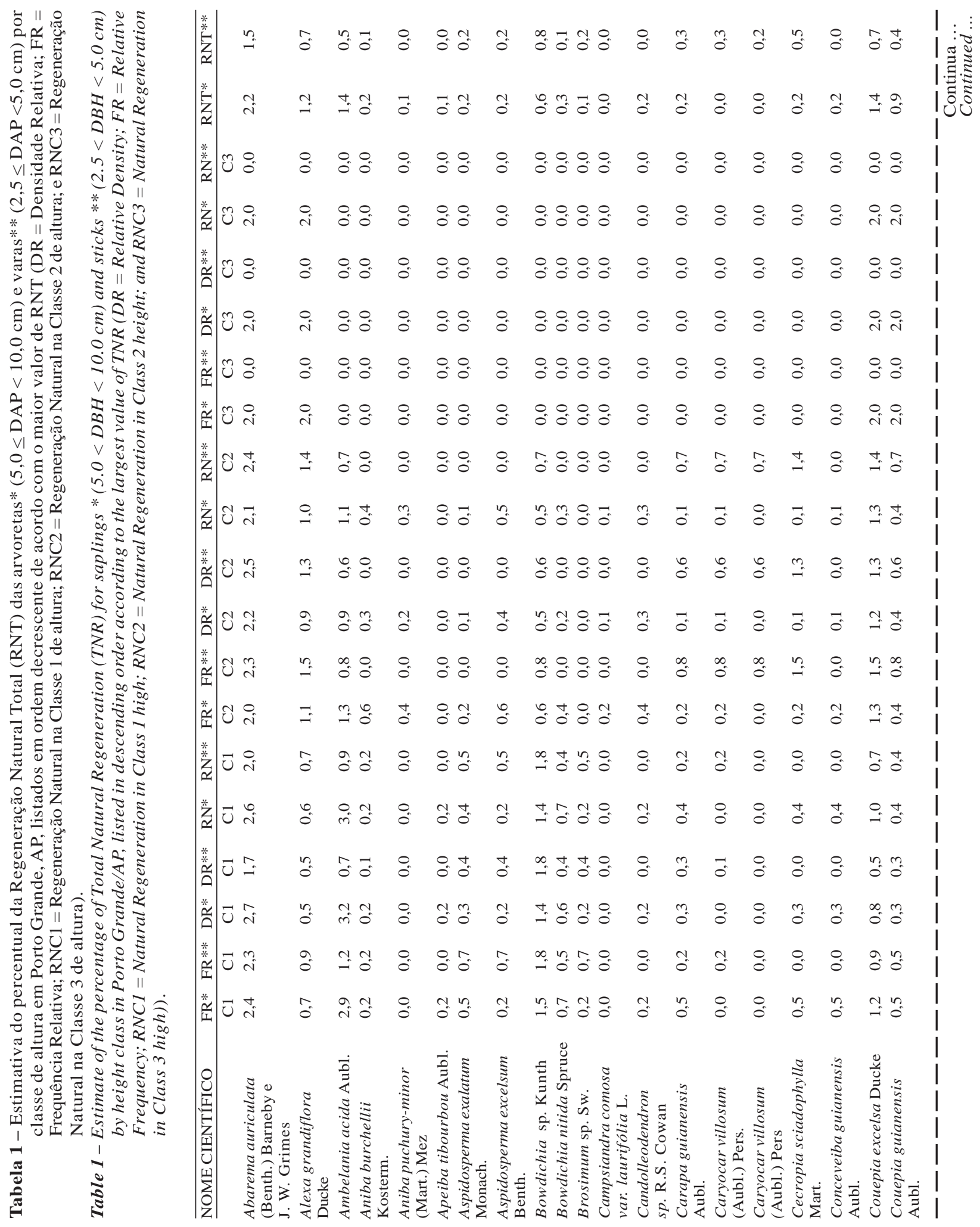




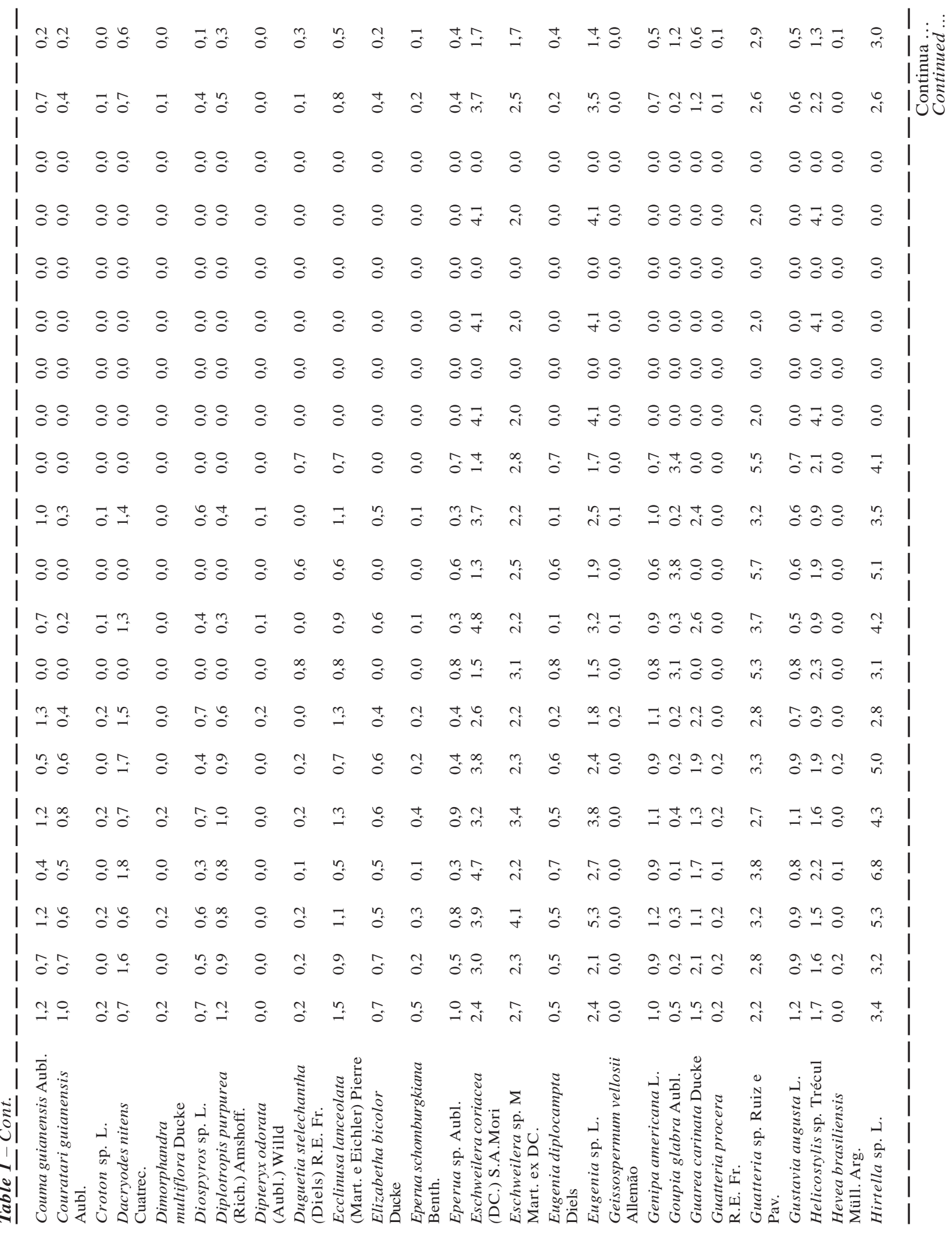




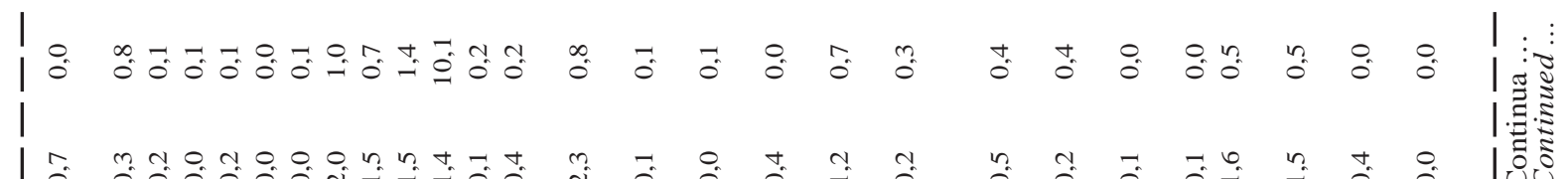

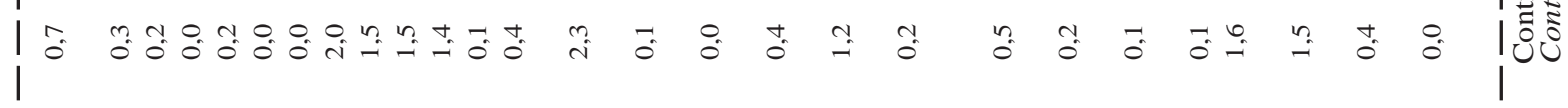

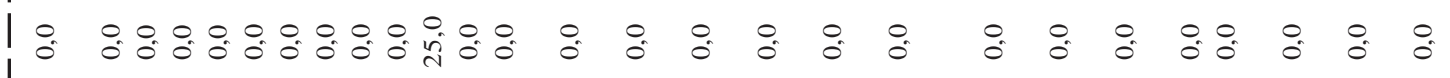

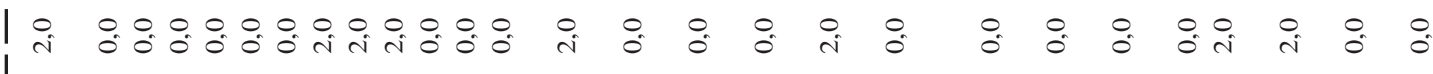

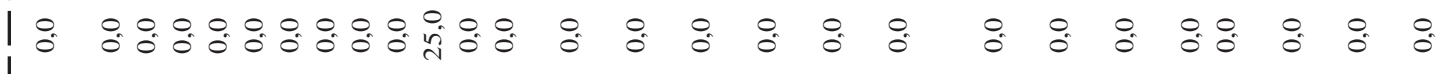

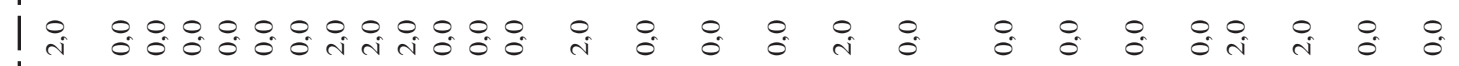

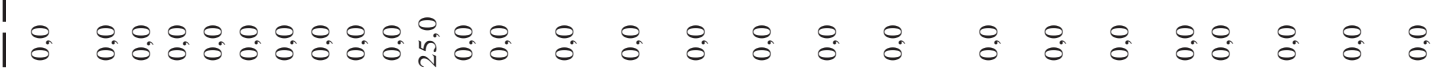
| | I: 0

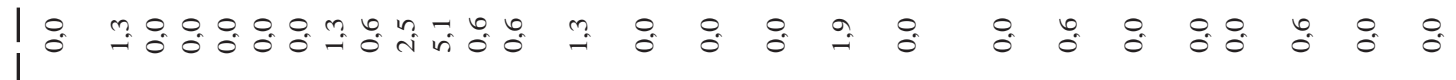

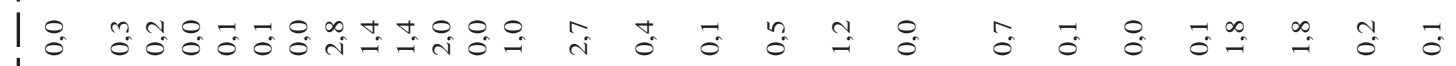

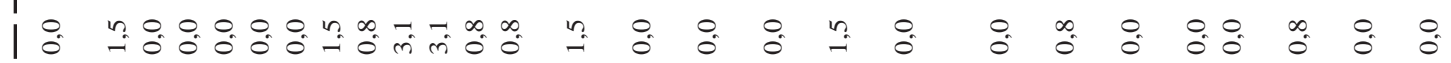

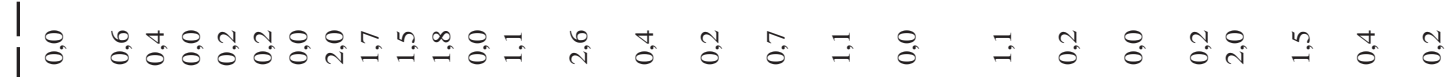
|

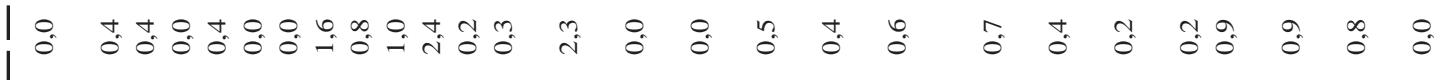

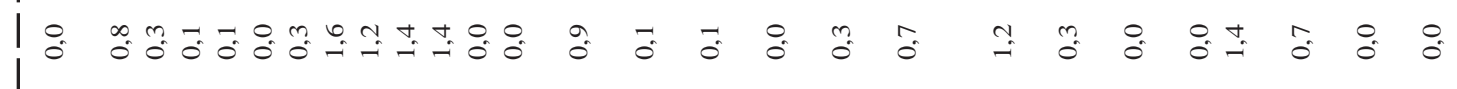

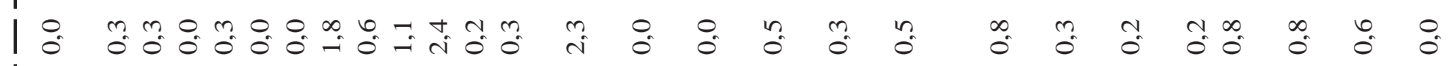

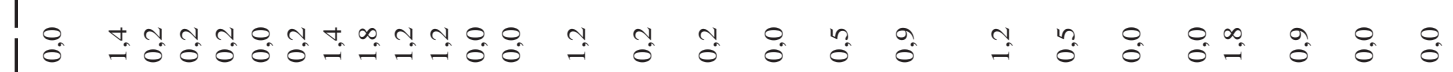

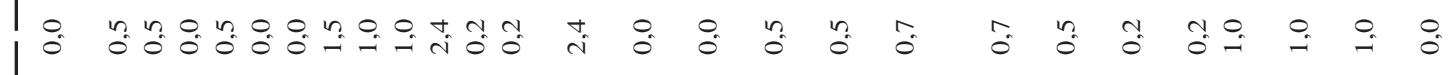

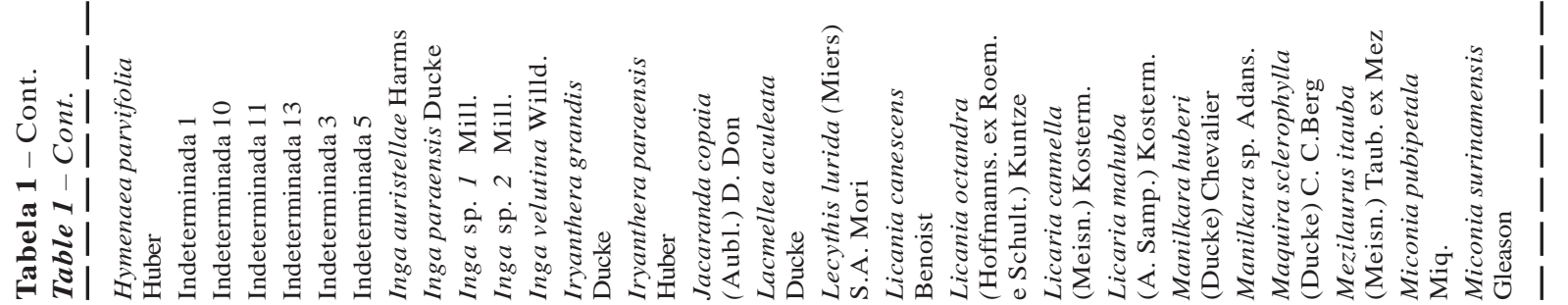




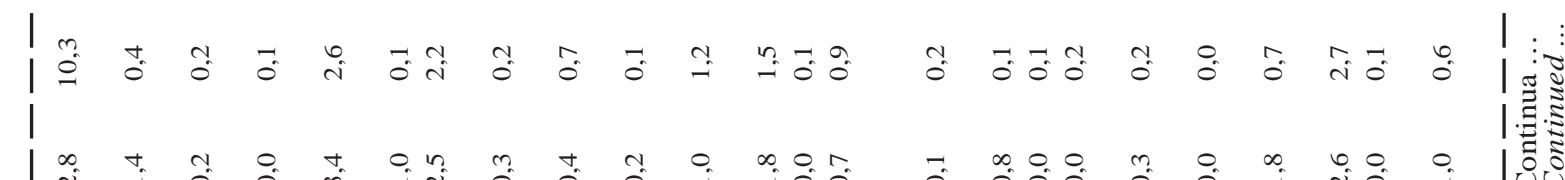

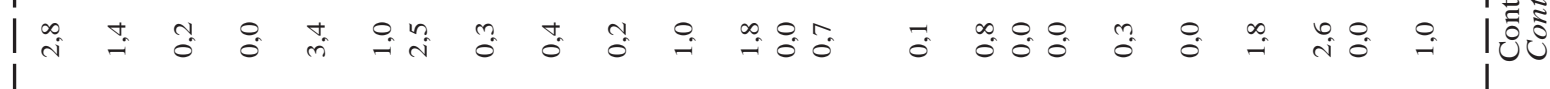

|

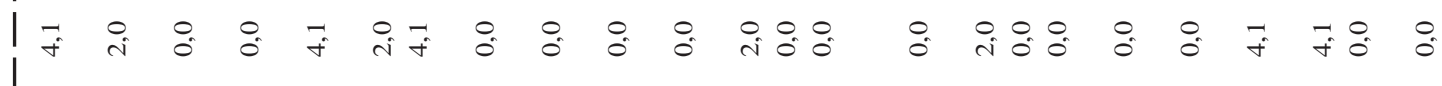

|

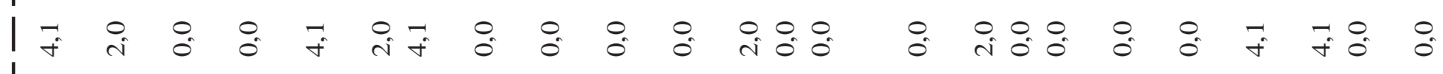

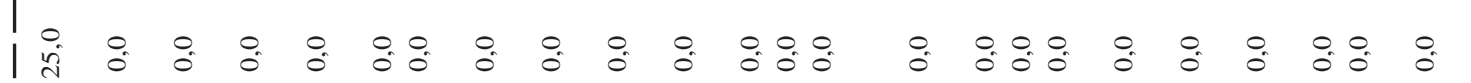

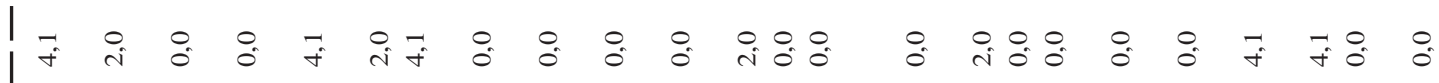

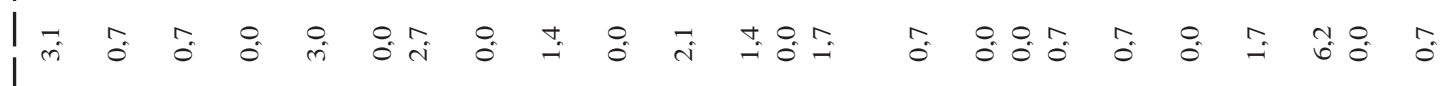

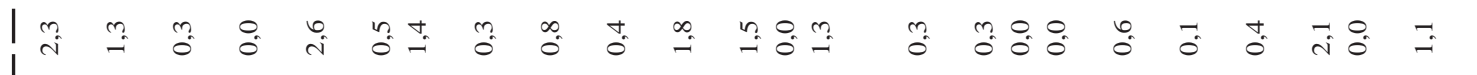

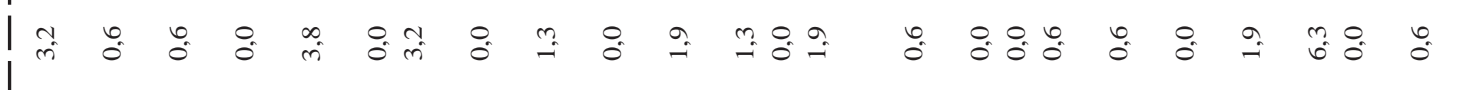

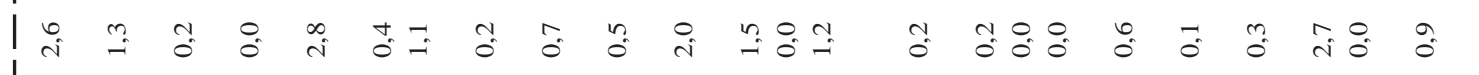

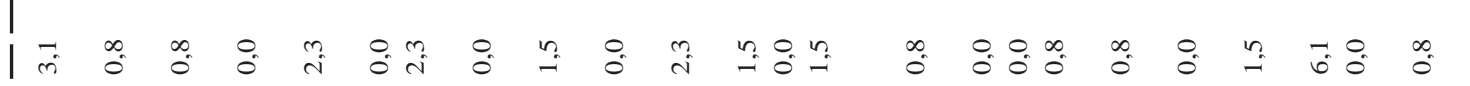

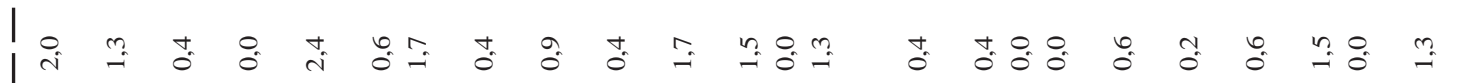

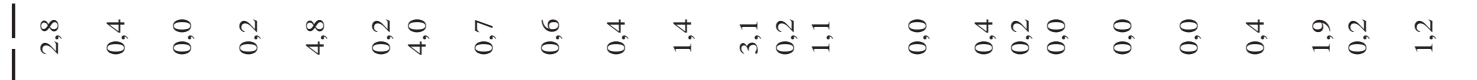

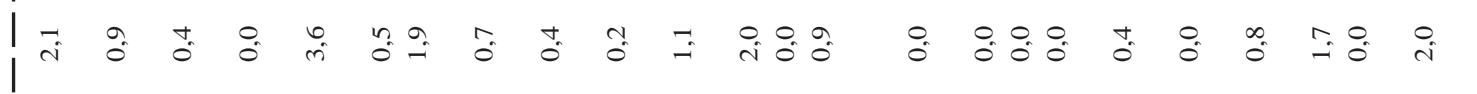

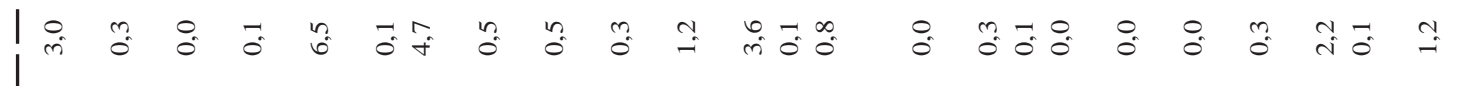

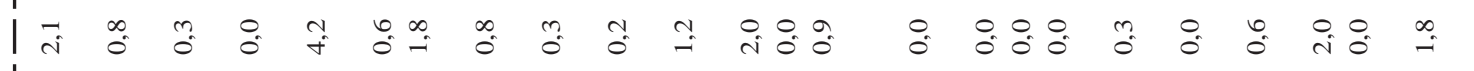

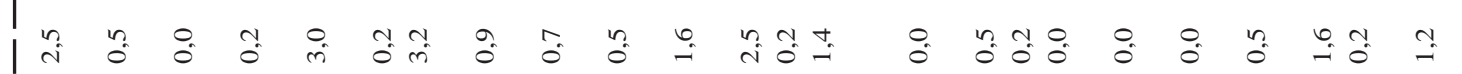

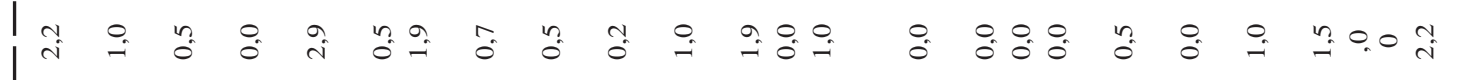

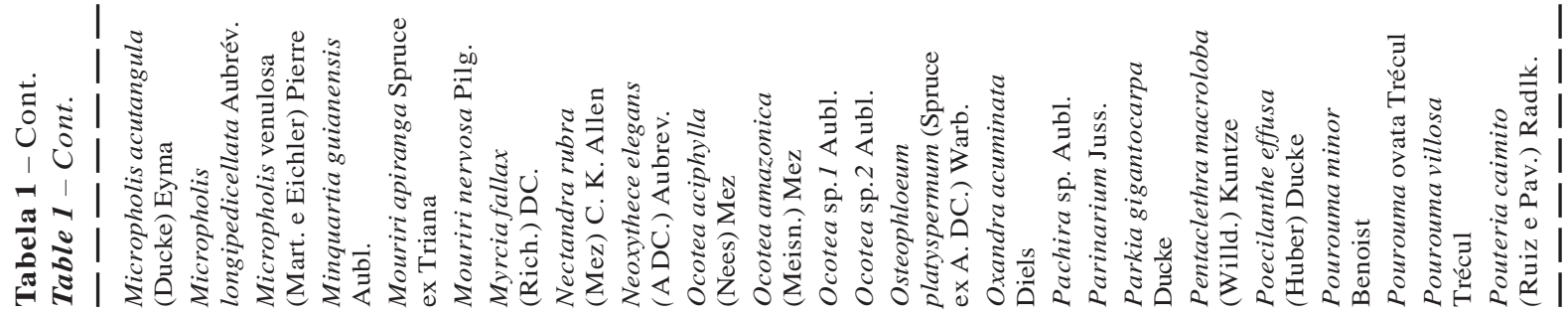




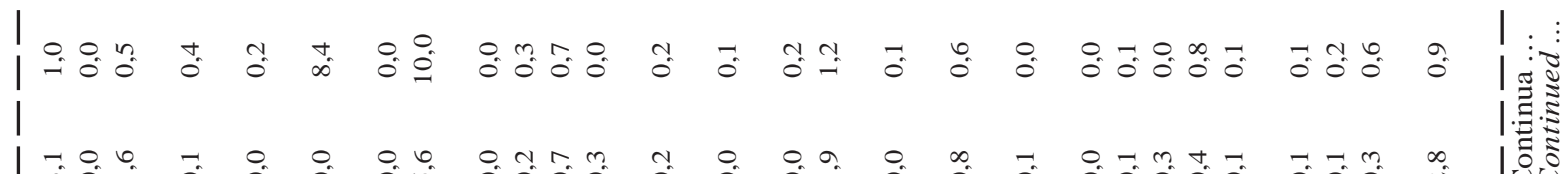

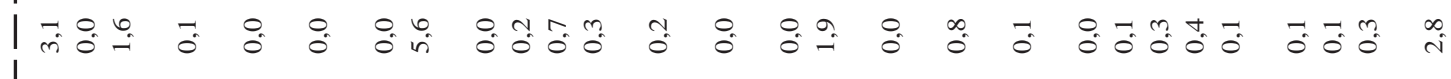

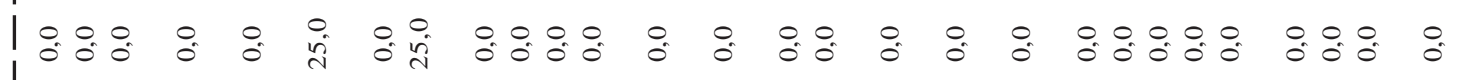

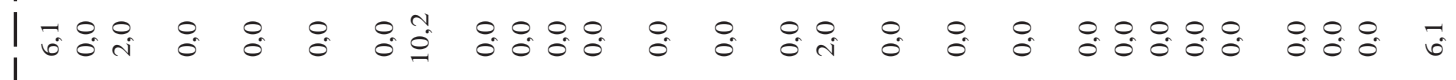

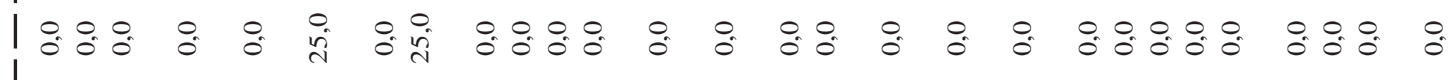

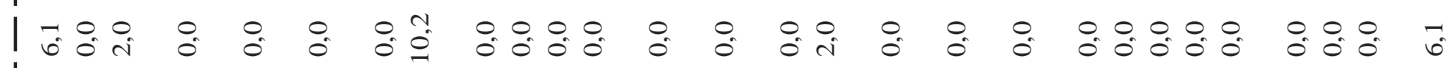

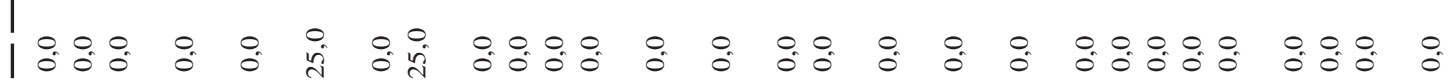

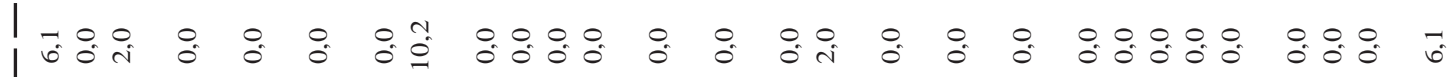

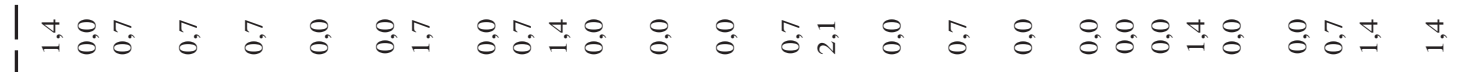
|

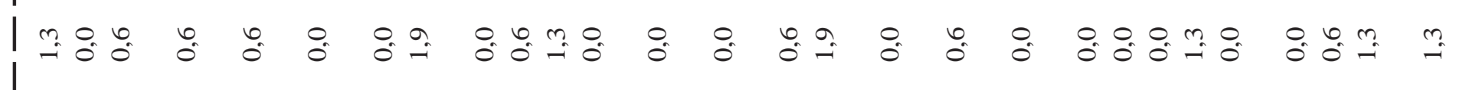

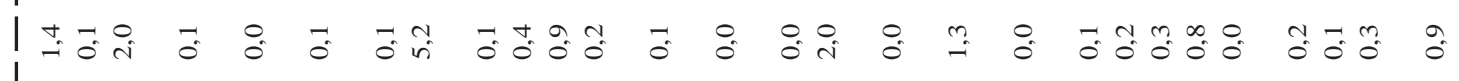

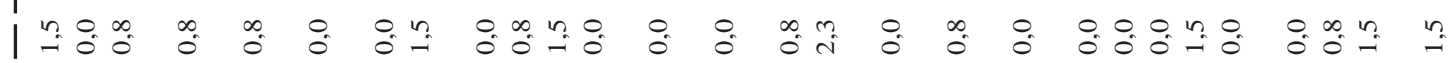

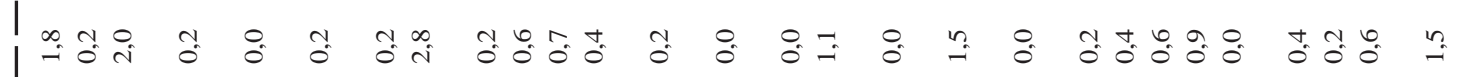
I

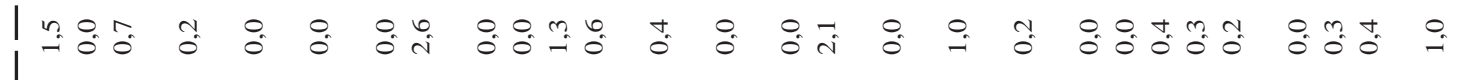
I

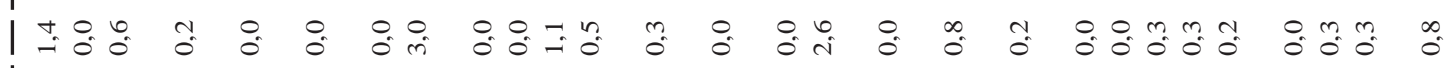

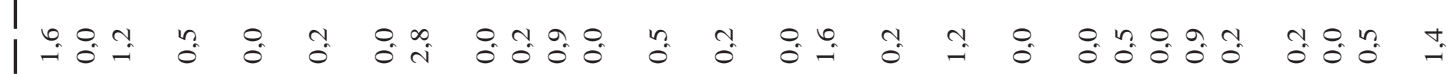
I

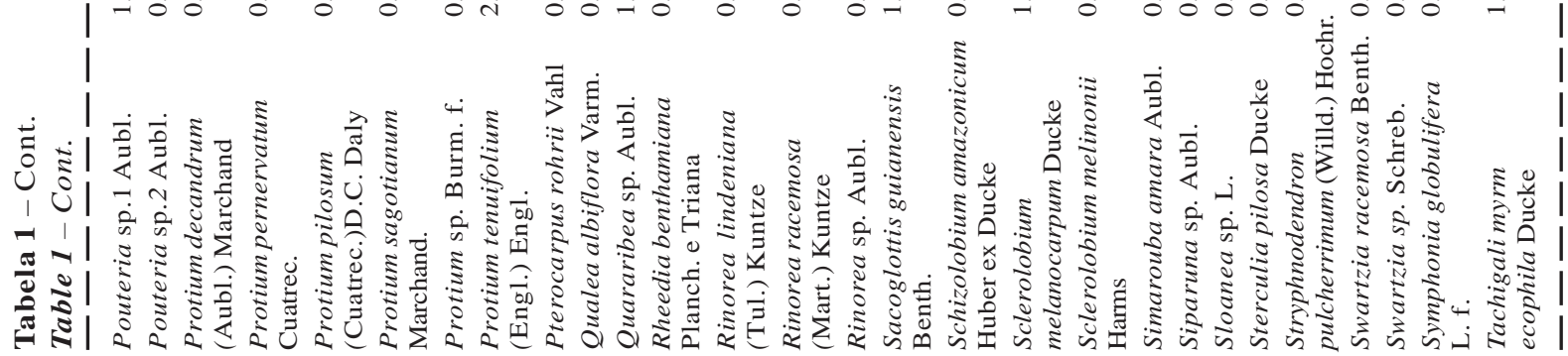




\begin{tabular}{|c|c|c|c|c|}
\hline 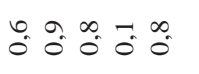 & $\overrightarrow{0}: 0$ & $\overrightarrow{0}$ & $\hat{0}:$ & $\begin{array}{cccc}m & N & = \\
0 & 0 & 0 & 0\end{array}$ \\
\hline $\begin{array}{lll}m & \Rightarrow & 0\end{array}$ & $\begin{array}{c}m \\
0\end{array}:$ & $\tilde{O}_{0}^{1}$ & $\therefore:$ & 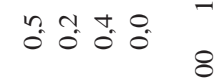 \\
\hline o & $\because: 0$ & : & $: 0$ & $\begin{array}{llll}0 & 0 & 0 & 0 \\
0 & 0 & 0\end{array}$ \\
\hline 000 & 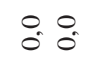 & : & is & $\begin{array}{lll}0 & 0 & 0 \\
0 & 0 & 0\end{array}$ \\
\hline 5000 & $0:$ & : & $0: 0$ & $\begin{array}{cccc}0 & 0 & 0 & 0 \\
0 & 0 & 0 & 0\end{array}$ \\
\hline $0_{0} 0^{\prime} 0^{\circ} 0^{\circ} 4$ & $\begin{array}{ll}0 \\
0\end{array}$ & : & $\begin{array}{c}0 \\
i\end{array}$ & $\begin{array}{cccc}0 & 0 & 0 & 0 \\
0 & 0 & 0 & 0 \\
0\end{array}$ \\
\hline $\begin{array}{llll}0 & 0 & 0 & 0 \\
0 & 0 & 0 & 0\end{array}$ & $\begin{array}{ll}0 & 0 \\
0 & 0\end{array}$ & : & $\begin{array}{ll}0 & 0 \\
0 & 0\end{array}$ & $\begin{array}{lll}0 & 0 & 0 \\
0 & 0 & 0\end{array}$ \\
\hline $\begin{array}{lll}0 & 0 & 0 \\
0 & 0 & 0\end{array}$ & $0: 0$ & : & $\stackrel{i}{i}$ & 00 \\
\hline 8 & $0: 0$ & : & $\stackrel{+}{-}$ & $\hat{0} 0$ \\
\hline$b$ & tas & : & $\rightarrow$ & $\begin{array}{l}0 \\
0 \\
0\end{array}$ \\
\hline $\begin{array}{lll}0 & 0 & 0 \\
0 & 0 & 0\end{array}$ & 0 & : & $m=$ & $\begin{array}{ll}0 & 0 \\
0 & 0\end{array}$ \\
\hline 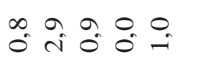 & $a_{0}^{m}=$ & $=$ & $\Rightarrow: 0$ & 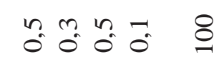 \\
\hline 0 & $0: 0$ & : & & $\begin{array}{llll}\infty & 0 & 0 & 0 \\
0 & 0 & 0 & 0 \\
0 & 0 & 0\end{array}$ \\
\hline 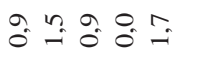 & $\begin{array}{ll}0 & N \\
0 & 0\end{array}$ & $\stackrel{2}{0}$ & $\stackrel{n}{\rightarrow}: 0$ & $\hat{n}=0$ \\
\hline 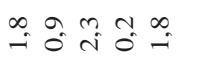 & $\begin{array}{ll}0 \\
0\end{array}$ & $\overbrace{0}^{2}$ & $\therefore$ & 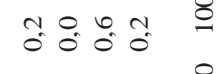 \\
\hline 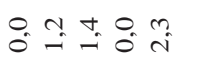 & $\begin{array}{ll}0 & 0 \\
0 & 0\end{array}$ & $\stackrel{0}{0}$ & 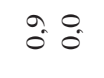 & $\begin{array}{l}0 . \\
0 \\
0\end{array}$ \\
\hline & to & is & $\infty$ & $\begin{array}{ll}m & 0 \\
0 & 0\end{array}$ \\
\hline$\overbrace{\rightarrow}^{n}$ & 농 & $m_{0}^{m}$ & $\begin{array}{l}0 \\
0 \\
0\end{array}$ & 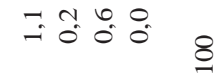 \\
\hline 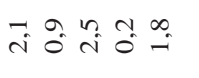 & $\begin{array}{ll}n & 0 \\
0 & 0 \\
0\end{array}$ & $\stackrel{1}{0}$ & $\begin{array}{l}0 \\
0 \\
0\end{array}$ & $\begin{array}{ccc}N & 0 & N \\
0 & 0 & 0 \\
0 & 0 & 0\end{array}$ \\
\hline 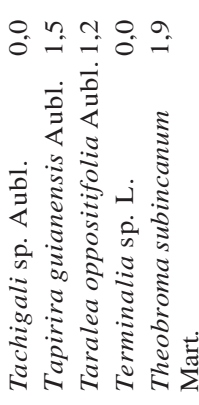 & 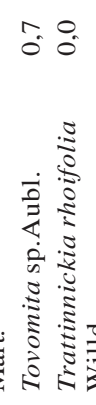 & 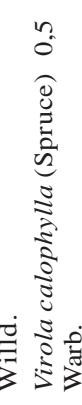 & 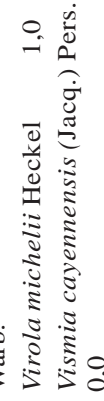 & 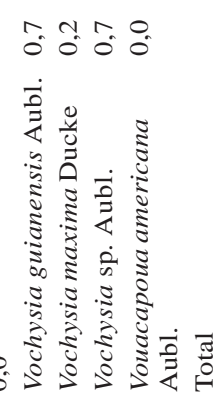 \\
\hline
\end{tabular}

Entre as espécies inventariadas em nível de vara, apenas 31 estavam presentes em todas as classes de regeneração natural. As espécies em nível de vara predominantes nas três classes de regeneração natural foram: Mouriri apiranga e Hirtella sp. (classe I), Guatteria sp. e Pourouma ovata (classe II) e Protium tenuifolium, Micropholis acutangula e Inga sp. 2 (classe III).

Quanto à regeneração natural total (RNT) na comunidade, as maiores variações foram encontradas nas espécies em nível de vara (10,3 a 0,1\%), enquanto as arvoretas pouco variaram (5,6 a 0,1\%). O número de espécies com percentuais inferiores a 1\% é elevado, com 121 e 103 espécies para varas e arvoretas, respectivamente, podendo-se citar as Indeterminadas, Vouacapoua americana, Carapa guianensis, Virola calophylla e Manilkara huberi.

Para a similaridade florística entre as arvoretas e varas nos distintos conglomerados, adotou-se como limite de significância a linha de fenon, a aproximadamente $50 \%$ da distância de ligação $(6,75)$. Nesse limite, houve a interceptação em três ramos do agrupamento, resultando em três grupos florísticos distintos (Figura 2).

Na formação dos grupos florísticos, foi observado que dois conglomerados apresentaram similaridade entre si. Entretanto, o primeiro grupo florístico contemplou

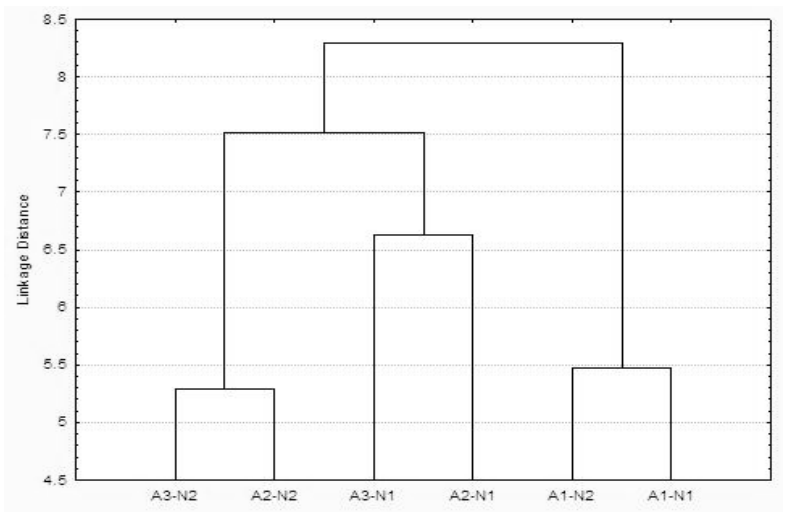

Figura 2 - Dendrograma representando as sequências de agrupamento em três áreas de estudo (A1; A2; A3) e dois níveis de regeneração natural (arvoreta $5.0 \leq$ dap $<10.0 \mathrm{~cm}$; vara $-2.5 \leq$ dap $<5.0 \mathrm{~cm}$ ) em floresta ombrófila densa, em Porto Grande, AP.

Figure 2-Dendrogram representing the grouping sequences in three study areas (A1, A2, A3) and two levels of natural regeneration (sapling $-5.0<d b h<$ $10.0 \mathrm{~cm}$; Stick - $2.5<d b h<5.0 \mathrm{~cm}$ ) in dense ombrophilous forest, Porto Grande, AP.

Revista Árvore, Viçosa-MG, v.38, n.4, p.699-710, 2014 
as espécies em nível de arvoreta nos dois conglomerados, enquanto o segundo agrupou, os indivíduos mais jovens (varas).

O conglomerado mais isolado formou o terceiro grupo da análise, em que se verificou similaridade da composição florística desde as plantas em nível de vara até os indivíduos mais bem estabelecidos (arvoreta).

No entanto, os grupos gerados apresentam distintas significâncias de formação, mensuradas por meio da proximidade do grupo com a linha de fenon. Tal característica (grupo x linha de fenon) sugere maior fragilidade de constituição dos grupos. O segundo grupo florístico apresentou a distância de ligação mais próxima da linha de fenon $(6,60)$, seguido do terceiro $(5,5)$ e do primeiro $(5,30)$.

\section{DISCUSSÃO}

Os valores dos índices de diversidade de Shannon encontrados (4,21 e 4,11 nats ind. $\left.{ }^{-1}\right)$ nos níveis de regeneração natural são considerados altos. No entanto, devem-se levar em consideração as diferenças nos estágios de sucessão, discrepâncias metodológicas e identificações taxonômicas, as quais variam nos levantamentos florísticos da região. Essa afirmação é baseada na variabilidade das diversidades usualmente encontradas em florestas tropicais (entre 1,5 e 3,5 nats.ind. ${ }^{-1}$ ), onde raramente ultrapassam 4,5 (DIAS et al., 2000; MARANGON et al., 2003; SILVA, 2007).

Em floresta ombrófila densa, Aparício et al. (2011b), analisando a regeneração natural de espécies arbóreas tropicais, obtiveram diversidade de Shannon de 3,32 nats ind. ${ }^{-1}$. Resultados semelhantes foram expostos em florestas secundárias no Pará, na avaliação momentânea da composição florística em regeneração (3,5 nats ind. $\left.{ }^{-1}\right)$ (RAYOL et al., 2008).

As espécies inventariadas com maior número de indivíduos são consideradas mais aptas a se estabelecer nas condições impostas pelo meio. Mantêm uma proporção de plantas entre os níveis de vara para arvoreta. No entanto, foi observado que os parâmetros de frequência e densidade das espécies variaram em função do nível de regeneração. Acredita-se que a variação pode estar relacionada ao tamanho das classes de altura que foram preestabelecidas para o estudo e para o estágio sucessional em que se encontrava a área.
As espécies que ocorrem nas três classes de altura de regeneração natural são aquelas que teoricamente possuem maior potencial de estabelecimento na floresta e que deverão estar presentes no futuro dossel (CITADINI-ZANETTE, 1995; SILVA, 2007). Entretanto, algumas espécies que apresentaram maiores índices de regeneração natural em nível de vara não foram observadas nas arvoretas, como o Protium sagotianum, que quando jovem foi classificado entre as espécies mais adaptadas, mas poucos indivíduos foram registrados na classe de tamanho imediatamente superior. Além disso, houve espécies encontradas apenas em uma classe de tamanho, apresentando regeneração natural baixa. Tal fato indica dificuldades no processo de estabelecimento, possivelmente ocasionado pela seletividade a que estão expostas ou pela dinâmica de germinação do banco de sementes.

A presença de uma espécie apenas na classe de menor altura (RNC1), com densidade elevada, pode apresentar valores de regeneração natural total (RNT) maior do que a de outras espécies presentes em todas as classes de altura (SILVA JUNIOR et al., 2008). Porém, de acordo com Volpato (1994), esse resultado deve ser analisado com cautela, já que a espécie pode desaparecer ainda nessa fase inicial de desenvolvimento.

Os baixos valores estimados de regeneração para algumas espécies na área de estudo também podem ser atribuídos à grande diversidade da área, à exposição da vegetação à declividade do terreno, do solo e do grupo ecológico e, principalmente, ao fato de a área ter poucos indícios antrópicos.

De modo geral, as espécies arbóreas em regeneração demonstraram potencial para autorrecuperação com diversidade específica elevada para as arvoretas e varas e com número de indivíduos significativo para compor o futuro dossel, deixando, assim, clara visão de um processo sucessional natural estabelecido para atingir uma floresta madura.

Em relação à similaridade florística, observou-se que as condições do estado de conservação das áreas são semelhantes, fortalecendo a permanência de exemplares florísticos específicos em cada fase da vegetação. No entanto, merece destaque o terceiro grupo florístico, formado pelo agrupamento de varas e arvoretas numa mesma área, demonstrando que o processo de sucessão está ocorrendo com perpetuação das espécies com maior índice de regeneração. 


\section{CONCLUSÃO}

As espécies arbóreas em regeneração demonstraram potencial para a autorrecuperação, com diversidade elevada nos níveis estudados e número de indivíduos capazes de compor o futuro dossel da floresta.

Estima-se que a estrutura e as relações entre os estratos verticais das espécies arbóreas em regeneração natural são capazes de desenvolver o processo sucessional de forma eficiente, garantindo a conservação da fitofisionomia da região.

\section{REFERÊNCIAS}

APARÍCIO, W. C. S. Estrutura da vegetação em diferentes ambientes na Resex do Rio Cajari: Interações do solo-floresta e relações com a produção de castanha. 2011. 150f. Tese (Doutorado em Ciências Florestais) Universidade Federal Rural de Pernambuco, Recife, 2011a.

APARÍCIO, W. C. S. Estrutura da regeneração natural de espécies arbóreas em um fragmento de Mata Atlântica, Pernambuco. Revista

Brasileira de Ciências Agrárias, v.6, n.3, p.483-488, 2011b.

APG II. An update of the Angiosperm Phylogeny Group classification for the orders and families of flowering plants: APG II. Botanical Journal of the Linnean Society, v.141, n.4, p.399-436, 2003.

CITADINI-ZANETTE, V. Fitossociologia e aspectos dinâmicos de um remanescente da Mata Atlântica na microbacia do Rio Novo, Orleans, SC. 1995. 236f. Tese (Doutorado em Ecologia e Recursos Naturais) - Universidade Federal de São Carlos, São Carlos, 1995.

DIAS, A. C.; CUSTÓDIO FILHO, A.; FRANCO, G. A. D. C. Diversidade do componente arbóreo, em floresta pluvial Atlântica secundária, São Paulo, Brasil. Revista do Instituto Florestal de São Paulo, v.12, n.2, p.127-153, 2000.

FINOL, U. H. Nuevos parametros a considerarse en el analisis estrutural de las selvas virgenes tropicales. Revista Forestal Venezolana, v.18, n.12, p.29-42, 1971.
GAMA, J. R. V. et al. Estrutura e potencial futuro de utilização da regeneração natural de Floresta de Várzea Alta no município de Afuá, estado do Pará. Ciência Florestal, v.13, n.2, p. 71-82, 2003.

INSTITUTO BRASILEIRO DE GEOGRAFIA E ESTATÍSTICA - IBGE. Manual técnico da vegetação brasileira. Rio de Janeiro: 2012.

LIMA FILHO, D.A. et al. Inventário florístico de floresta ombrófila densa de terra firme, na região do Rio Urucu-Amazonas, Brasil. Acta

Amazonica, v.31, n. 4, p.565-579, 2001.

MARANGON, L. C.; SOARES, J. J.; FELICIANO, A. L. P. Florística arbórea da mata da pedreira, município de Viçosa, Minas Gerais. Revista Árvore, v.27, n.2, p.207-215, 2003.

MARTINS, S. V.; RODRIGUES, R. R. Gap-phase regeneration in a semideciduous mesophytic forest, south-eastern Brazil. Plant Ecology, v.163, v.1, p.51-62, 2002.

ODUM, E. P. Fundamentos de ecologia. 4.ed. Lisboa: Fundação Calouste Gulbenkian, 1988. 927p.

PRANCE, G. T.; RODRIGUES, W. A.; SILVA, M. F. Inventário florestal de um hectare de mata de terra firme, km 30 da estrada Manaus-Itacoatiara. Acta Amazonica, v.6, n.1, p.9-35, 1976.

RADAM. Projeto RadamBrasil. Folha SB22 Araguaia e parte da folha SC 22 Tocantins. Rio de Janeiro: Instituto Brasileiro de Geografia e Estatística,1974.

RAYOL, B. P.; ALVINO, F. O.; SILVA, F. F.

Estrutura e composição florística da regeneração natural de duas florestas secundárias em Capitão Poço, Pará, Brasil. Amazônia: Ciência \& Desenvolvimento, v.4, n.7, p. 103-116, 2008.

SILVA JUNIOR, J. F. et al. Estudo fitossociológico de espécies florestais arbóreas em um fragmento de Floresta Atlântcia no Cabo Santo Agostinho PE. Ciências Agrárias e Ambientais, v.1, p.1, 2008.

SILVA, J. N. M. et al. Diretrizes para instalação e medição de parcelas permanentes em florestas naturais da Amazônia Brasileira. Belém: 2005.

Revista Árvore, Viçosa-MG, v.38, n.4, p.699-710, 2014 
SILVA, W. C. et al. Estudo da regeneração natural de espécies arbóreas em fragmento Floresta Ombrófila Densa, Mata das Galinhas, no Município de Catende, Zona da Mata Sul de Pernambuco. Revista Ciência Florestal, v.17, n.4, p.321-331, 2007.
VOLPATO, M. M. L. Regeneração natural em uma floresta secundária no domínio de Mata Atlântica: uma análise fitossociológica. 1994. 123f. Dissertação (Mestrado em Ciência Florestal) Universidade Federal de Viçosa, Viçosa, MG, 1994. 